Case Report

\title{
Central Diabetes Insipidus in a Patient with Stiff Person Syndrome
}

\author{
Fatima Zarzour, Abir Bou Khalil, Sami Toufic Azar* \\ Department of Endocrinology and Metabolism, American University of Beirut Medical Center, Beirut, Lebanon
}

Email address:

sazar@aub.edu.lb (S. T. Azar)

${ }^{*}$ Corresponding author

\section{To cite this article:}

Fatima Zarzour, Abir Bou Khalil, Sami Toufic Azar. Central Diabetes Insipidus in a Patient with Stiff Person Syndrome. American Journal of Internal Medicine. Vol. 7, No. 6, 2019, pp. 151-153. doi: 10.11648/j.ajim.20190706.13

Received: August 14, 2019; Accepted: September 6, 2019; Published: November 19, 2019

\begin{abstract}
Background: Central diabetes insipidus (CDI) is a rare hypothalamic-pituitary disease due to the deficiency of arginine vasopressin (AVP) synthesis of which more than one third of etiologies are unidentified. Autoimmunity is associated with one third of patients with apparently idiopathic CDI. The most common antibody detected in auto immune CDI is autoantibodies to AVP-secreting cells (AVPcAb). Stiff-person syndrome (SPS) is a rare neurological disorder with features of an autoimmune disease. It is characterized by fluctuating muscle rigidity in the trunk and limbs and a heightened sensitivity to stimuli. It is well known that SPS is associated with multiple auto immune endocrinopathy including auto immune diabetes and auto immune thyroid disease. The association between SPS and CDI is not well documented in the literature. Case information: We report a case of 51 year old female who developed CDI while being treated for SPS unmasked by high dose steroids. Result: Anti amphiphysin is the only antibody detected in our patient. Animal studies showed a high expression of amphiphysin in anterior and posterior pituitary gland, data in human are still vague .One theory is that anti-amphiphysin antibodies attack the amphiphysin molecules in the AVP secreting cells and inhibit release of AVP. Conclusion: This antibody could hint towards the autoimmune cause of CDI.
\end{abstract}

Keywords: Central Diabetes Insipidus, Stiff Person Syndrome, Anti Amphyphysin Antibodies

\section{Introduction}

CDI is a rare hypothalamic-pituitary disease due to the deficiency of AVP synthesis from the hypothalamus and/or secretion from the neurohypophysis. The etiology of CDI is unknown in over one third of cases, and thus it is classified as idiopathic CDI. We report a case of CDI associated SPS.

\section{Case Report}

This is a case of 50 year-old lady, previously healthy, who presented for recurrent spells of whole body shaking. These episodes occurred for 2 months, on a daily basis, triggered by fear, urination or defecation. The symptoms were associated with bilateral wrists spasm, progressive weakness until the patient became bedridden.

General physical exam was unremarkable. On neurological exam, cranial nerves were intact, prominent $4 / 5$ motor power on the left side, compared to $5 / 5$ on the right side, with a dystonic posture of the left wrist in an extended position. She had $4+$ reflexes in bilateral lower extremities, ankle clonus bilaterally and down-going toes. She was admitted initially without clear diagnosis for further workup.

During her hospitalization, the patient had an increased frequency of her whole body shaking episodes. On EEG, there was no seizure activity and the same spells were induced and reproduced by hyperventilation. Then she got intubated for airway protection.

Her CSF results were consistent with lymphocytic pleiocytosis but a negative infectious workup.

Auto-antibodies were ordered, in the suspicion of auto-immune and/or paraneoplastic encephalomyelitis. All antibodies were negative except for anti-amphiphysin (associated with paraneoplastic conditions or SPS). PET scan 
was negative for malignancy. In the repeat CSF studies, oligoclonal bands were positive, and the patient was diagnosed with SPS. She was given pulse steroids for 7 days without improvement. A pan CT was ordered for a new disease evaluation looking for primary neoplasm. Thyroid nodules were biopsied and were benign. Left axillary lymph node was also biopsied and revealed metastatic ductal carcinoma with breast as origin. After repeated extensive discussion, the family refused surgical option (mastectomy).

After starting pulse steroids, and while intubated in ICU, the patient started to have polyuria: urine output increased to 7 liters per day with a net negative balance of 5 liters. Symptoms were associated with hypernatremia. The trend of sodium over the days of hospitalization showed a high normal sodium level even before steroids initiation (table 1).

Table 1. Sodium, serum osmolality, urine osmolality and urine sodium levels before and after treatment with desmopressin.

\begin{tabular}{llllll}
\hline & $\mathbf{1 0} / \mathbf{1 0} / \mathbf{2 0 1 8}$ & $\mathbf{1 1 / 1 0 / 2 0 1 8}$ & $\mathbf{1 2} / \mathbf{1 0} / \mathbf{2 0 1 8}$ & $\mathbf{1 3 / 1 0 / 2 0 1 8}$ & $\mathbf{1 6 / 1 0 / 2 0 1 8}$ \\
\hline Sodium & 147 & 147 & 145 & 144 & 139 \\
Serum osmolality & 300 & & 295 & 289 & 284 \\
Urine osmolality & 96 & 306 & 599 & 95 & 615 \\
Urine sodium & 117 & 241 & 249 & & 96 \\
\hline
\end{tabular}

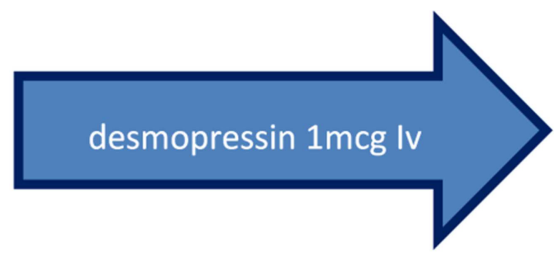

Figure 1. Correction of hypernatremia with initiation of desmopressin.

Patient was diagnosed with diabetes insipidus based on the inability to concentrate urine in the setting of hypernatremia. The increase of urine osmolality more than $100 \%$ after desmopressin administration pointed towards CDI. Water deprivation test was not done since patient was in critical care unit. Patient was then switched from IV to sublingual desmopressin to maintain normonatremia and prevent polyuria. MRI sella showed normal pituitary gland with normal pituitary stalk enhancement. Cortisol, TSH, FT4 were normal. Auto-immune endocrinological work up including anti GAD, anti TPO and anti TG were normal.

\section{Discussion}

DI is part of a group of hereditary or acquired polyuria and polydipsia diseases. It is associated with inadequate AVP or antidiuretic hormone (ADH) secretion or renal response to AVP, resulting in hypotonic polyuria and a compensatory/underlying polydipsia [1]. The AVP system maintains water balance based on serum osmolality and arterial blood volume via the vasopressin-2-receptor (V2R) [2]. CDI is caused by inadequate synthesis/release of AVP, often secondary to surgery or head injury which causes traumatic injury to the hypothalamus or posterior pituitary gland and destruction/degeneration of neurons originating in the supraoptic and paraventricular nuclei of the hypothalamus [3]. The most common and well recognized causes include local inflammatory or autoimmune diseases, vascular disorders, Langerhans cell histiocytosis (LCH), sarcoidosis, tumors such as germinoma/craniopharyngioma or metastases, traumatic brain injuries, intracranial surgery, and midline cerebral and cranial malformations [4]. Rosario et al found that autoimmunity is associated with one third of patients with apparently idiopathic CDI, which should therefore be classified as autoimmune CDI. Autoimmune CDI is highly likely in young patients with a clinical history of autoimmune diseases and radiological evidence of pituitary stalk thickening. Similarly a recent article mentions that autoimmune destruction of the neurohypophysis is the most likely cause of many cases of idiopathic central DI which were proven to be autoimmune through biopsy samples, post- mortem examination and high prevalence of anti- vasopressin cell antibodies [5]. Rosario et al reported that autoantibodies to AVP-secreting cells (AVPcAb) were negative in $17.2 \%$ of idiopathic CDI even with presence of other autoimmune manifestations (presence of autoimmune thyroid diseases or type I diabetes mellitus and/or thyroid and islet cell autoantibodies). The most common antibody is AVPcAb. Maghnie et al showed that AVPc-Abs were present in eight out of nine patients with idiopathic CDI and pituitary stalk thickening either at the time of the first neuroimaging evaluation or at the follow-up [6]. Their presence was strongly associated with female sex, duration of the disease, age less than 30 years, absence of neurohypophyseal bright spot and presence of pituitary stalk thickening [7]. Early work by Scherbaum and Bottazzo demonstrated positive AVP cell antibodies in one-third of a cohort of patients with idio-pathic DI recruited from a number of academic centres in London. They later reported rates of overt autoimmune disease in $28 \%$ of a similar cohort, and similar rates were subsequently confirmed by other groups; pernicious anemia and thyroid disease are most common [8].

The only antibody detected in our patient was anti-amphiphysin antibody. Amphiphysin is a 125 to $128 \mathrm{KDa}$ cytoplasmic synaptic vesicle associated protein expressed in neurons, certain types of endocrine cells and spermatocytes. Its biological action involve synaptic vesicle endocytosis. The pathophysiology of SPS involves the impairment of GABAergic neurotransmission. While 6 separate autoantigens have been identified [9], the presence of anti-GAD antibodies has been reported in $43 \%$ [9] to $85 \%$ [10] of patients. Amphiphysin antibodies are present in SPS patients negative for GAD antibodies, all of whom are female breast cancer patients [11]. The expression of amphiphysn in posterior pituitary cells in human is still vague. One theory is 
that anti-amphiphysin antibodies attack the amphiphysin molecules in the AVP secreting cells and inhibit release of AVP. Animal studies showed a high expression of amphiphysin in anterior and posterior pituitary gland. Beate Lichte and her colleagues showed that this protein is expressed in the brain, adrenal gland, anterior pituitary and posterior pituitary of rats and chicken [12]. While this is hard to prove, other anterior pituitary hormones deficiency were reported in patient with defect in pathways involving amphiphysin. Birgitte Holst et al, identified the lipid binding BAR (Bin/amphiphysin/Rvs) domain protein PICK1 (protein interacting with $\mathrm{C}$ kinase 1) as a key component early in the biogenesis of secretory vesicles in GH-producing cells. Defect in this pathway was associated with severe GH deficiency [13]. Qiao et al, showed that CDI was found in 4 out of 13 patients with positive antiamphiphysin antibodies associated with paraneoplastic syndrome [14].

In addition, cortisol induces a form of relative AVP resistance by interfering with AVP signaling at the V2 receptor or post-receptor level leading to increased free water excretion and unmasking of CDI [15].

In our patient, the presence of amphiphysin antibodies may have led to the inhibition of AVP release, leading to CDI which was further exacerbated by high dose steroid to the point requiring desmopressin treatment.

\section{Conclusion}

Although SPS is known to be associated with multiple endocrinopathy including type $1 \mathrm{DM}$ and auto immune thyroid disease, the data about association with autoimmune CDI is scarce. We reported a case of CDI in patient with SPS. The pathophysiology behind this association and whether anti amphiphysin antibodies are directly involved is yet not clear. More studies at the molecular level need to be conducted in this field.

\section{References}

[1] Malve H, Kalra S, Zargar A, Jain S, Sethi B, Chowdhury S, et al. Diabetes insipidus: The other diabetes. Indian Journal of Endocrinology and Metabolism. 2016; 20 (1): 9.

[2] Juul KV, Bichet DG, Nielsen S, Nørgaard JP. The physiological and pathophysiological functions of renal and extrarenal vasopressin V2 receptors. American Journal of Physiology-Renal Physiology. 2014; 306 (9).

[3] Iorgi ND, Napoli F, Allegri AEM, Olivieri I, Bertelli E, Gallizia
A, et al. Diabetes Insipidus - Diagnosis and Management. Hormone Research in Paediatrics. 2012; 77 (2): 69-84.

[4] Murdaca G, Russo R, Spanò F, Ferone D, Albertelli M, Schenone A, Contatore M, Guastalla A, De Bellis A, Garibotto G, Puppo F. Autoimmune central diabetes insipidus in a patient with ureaplasma urealyticum infection and review on new triggers of immune response. Arch Endocrinol Metab. 2015 Dec; 59 (6): 554-8.

[5] Christ-Crain M, Bichet D G, Fenske W K, Goldman M B, Rittig S, Verbalis J G, \& Verkman A S. Diabetes insipidus. Nature Reviews Disease Primers. 2019; 5 (1).

[6] Maghnie M, Ghirardello S, Bellis AD, Iorgi ND, Ambrosini L, Secco A, et al. Idiopathic central diabetes insipidus in children and young adults is commonly associated with vasopressin-cell antibodies and markers of autoimmunity. Clinical Endocrinology. 2006; 65 (4): 470-8.

[7] Pivonello R, Bellis AD, Faggiano A, Salle FD, Petretta M, Somma CD, et al. Central Diabetes Insipidus and Autoimmunity: Relationship between the Occurrence of Antibodies to Arginine Vasopressin-Secreting Cells and Clinical, Immunological, and Radiological Features in a Large Cohort of Patients with Central Diabetes Insipidus of Known and Unknown Etiology. The Journal of Clinical Endocrinology \& Metabolism. 2003; 88 (4): 1629-36.

[8] Garrahy A, Moran C, Thompson C et al. Diagnosis and management of central diabetes insipidus in adults. Clinical Endocrinology. 2019; 90: 23-30.

[9] Martinez-Hernandez E, Arino H, McKeon A, et al. Clinical and immunologic investigations in patients with stiff-person spectrum disorder. JAMA Neurol. 2016; 73: 714-20.

[10] Rathbun J, Imber J et al. Stiff Person Syndrome and Type 1 Diabetes Mellitus: a Case of the Chicken or the Egg? Journal of Genral Internal Medicine. 2019; 34 (6): 1053-10574.

[11] Robert L, Julius C et al. Atlas of immunology. 2004 (book).

[12] Beate L, Rudiger WV, Helmut E. M, Manfred WK. et al. Amphiphysin, a novel protein associated with synaptic vesicles. The EMBO Journal vol. 11 no. 7 pp. 2521-2530, 1992.

[13] Holst B, Madsen KL, Jansen AM, Jin C, Rickhag M, Lund VK, et al. PICK1 Deficiency Impairs Secretory Vesicle Biogenesis and Leads to Growth Retardation and Decreased Glucose Tolerance. PLoS Biology. 2013; 11 (4).

[14] L Qiaou. Et al. Paraneoplastic neurological syndromes associated with anti-amphiphysin antibodie. October 2016.

[15] Chin H, Quek T, Leow M. Central diabetes insipidus unmasked by corticosteroid therapy for cerebral metastases: beware the case with pituitary involvement and hypopituitarism. Journal of the Royal College of Physicians of Edinburgh. 2018; 47 (3): 247-9. 